From the Desk of the Edito in Chief

\title{
From the Desk of the Editor in Chief
}

It is a great pleasure and satisfaction that volume 6 issue 2 of Anwer Khan Modern Medical College Journal have been published in time. I am very thankful to the authors from both local and outside for their interest to publish article in Anwer Khan Modern Medical College Journal. 4 Original article, 3 Review article, 1 Case report and an Editorial have been included in this issue of Journal for publication.

Editorial on "Challenging medical education: A long way up to meet" focusses on importance on reducing the disease burden like malnutrition, communicable \& non communicable disease in Bangladesh through integrating these in medical curriculum and proper implementation.

Atticle on "Goldsmith workers (GSWS): A skilled job \& status of illness" reflects the magnitude of respiratory problem faced by workers in this industry the incidence of which is very high (37\%).

People specially residing in rural areas consume a lot of starfruit. It is a citrus rich fruit and of can cause Nephrotoxicity because of calcium oxalate crystal and apoptosis of renal epithelial cells leading to end stage renal diseases. Review article on this is a timely awareness paper.

Review Article on "platelet transfusion therapy" emphasis the need for prophylactic transfusion to reduce active bleeding risk of bleeding specially in patients with thrombocytopenia, intensive chemotherapy and bone marrow transplantation

Another review paper highlighting Human resource request under the context of "University health coverage (HC): Current situation and feature challenges" is a timely printed paper which focusses on how to achieve remarkable progress on the overall health status in Bangladesh.

Original article on "Peritoneal dialysis in children with acute kidney injury: Dhaka shishu (Children) Hospital experience concluded that PD can be successfully performed for management of child hood AKI. Septaecaemia and Hypovolumia are the leading causes of AKI, however mortality is higher in male with unclear etiology.

Original article on "Assessment of morbidity and mortality of thyroid surgery" concludes improved surgical techniques and proper management of complications reduce the post operative morbidity and mortality of thyroid surgery.

Original article on "Depression among nural aged: facts" and reasons states 5\% of the people aged over 60 suffers from depression. This indicates depression as common problem in aged which is not a normal part of ageing finding demand awareness among family members and comprehensive health care facility for the aged people.

Pattern of psychiatric illness among tuberculosis patients emphasises the need of psychiatric consultation for improvement of continuation and adherence to long-term treatment failure which is responsible for MDR tuberculosis in Bangladesh.

Case report on "An annual case of foreign body Aspiration" describes the importance of careful history, clinical examination along with bronchoscopy to identify and manage foreign body aspiration especially in children.

Overall all the articles will hopefully be interesting to the readers. 